\title{
ANÁLISIS MULTIVARIADO DE LA FERTILIDAD DE LOS SUELOS EN SISTEMAS DE CAFÉ ORGÁNICO EN PUENTE ABADIA, VILLAVICENCIO
}

\section{MULTIVARIATE ANALYSIS OF SOIL FERTILITY IN ORGANIC COFFEE SYSTEMS IN PUENTE ABADIA VILLAVICENCIO}

\author{
Amanda Silva-Parra ${ }^{1}$, Carlos Colmenares-Parra ${ }^{2}$, Jorge Álvarez-Alarcón ${ }^{3}$
}

\begin{abstract}
${ }^{1}$ Ing. Agrónoma, M.Sc., Ph.D., Profesora Asistente. Universidad de los Llanos, Villavicencio, Colombia, Km 12 Vía a Puerto López, Vereda Barcelona, C.P. 1745, e-mail: asilvap@unillanos.edu.co; ${ }^{2}$ Ing. agrónomo, M.Sc., Profesor Asistente. Universidad de Los Llanos, Villavicencio, Colombia, Km 12 Vía a Puerto López, Vereda Barcelona, C.P. 1745, e-mail: ccolmenares@ unillanos.edu.co; ${ }^{3}$ Estudiante Ing. Agronómica. Universidad de Los Llanos, Villavicencio, Colombia, km 12 Vía a Puerto López, Vereda Barcelona, C.P. 1745, e-mail: jorge.alvarez@unillanos.edu.co; Grupo de Investigación en Sistemas Agrícolas y Forestales ISAF, Facultad de Ciencias Agropecuarias y Recursos Naturales, Universidad de los Llanos.
\end{abstract}

Rev. U.D.C.A Act. \& Div. Cient. 20(2): 289-298, Julio-Diciembre, 2017

\section{RESUMEN}

Puente Abadía es una zona cafetera por excelencia, del municipio de Villavicencio, Departamento del Meta, Colombia, donde se cultivan sistemas de café orgánico (SCO). El manejo agronómico del cultivo puede influir en las propiedades químicas de sus suelos. El análisis multivariado permitirá definir algunas limitantes químicas de los suelos y será un insumo futuro importante, para optimizar el manejo de nutrientes. Se muestrearon en la zona 12 SCO, que presentaban similitud en cuanto a tipo de sistema de producción, manejo agronómico y condiciones ambientales. Los datos fueron analizados, a través de estadística descriptiva, correlaciones lineales y los métodos multivariados de clúster y análisis de componentes principales (ACPs). En los SCO, se confirmó que existe una alta heterogeneidad en las propiedades químicas de los suelos. El Mg presentó el coeficiente de variación (CV) más alto (135,09\%). El ACPs mostró que los dos primeros componentes principales explicaron más del $50 \%$ de la varianza total. El análisis de clúster de los SCO mostró alta heterogeneidad en las propiedades químicas de los suelos, conformándose dos grupos: el grupo 1 reunió a los SCO, dominados por una alta acidez intercambiable, incluyendo 3 de los 12 SCO y, el grupo 2, reunió a 9 de los SCO, definidos, principalmente, por altos contenidos de materia orgánica del suelo (MOS). La alta variabilidad encontrada fue influenciada, posiblemente, por el encalado y la fertilización orgánica.

Palabras clave: Acidez intercambiable, análisis de suelo, encalado, materia orgánica, propiedades químicas.
SUMMARY

Puente Abadía is for excellence a coffee growing area of the municipality of Villavicencio, department Meta, Colombia, where organic coffee systems OCS are cultivated. The agronomic management of the crop can influence its soil chemical properties. Multivariate analysis can permit to define some chemical soil limits and will be a future input to optimize the nutrient management. Twelve OCS were sampled in the zone that showed similarity in terms of production system, agronomic management and environmental conditions. Data were analyzed by descriptive statistics, linear correlations and multivariate clustered and principal components PC methods. High variability in OCS chemical properties were detected. The highest variation coefficient CV was presented in $\mathrm{Mg}$ 135.09\%. The analysis of the principal components (APCs) showed that the first two PC explained more than $50 \%$ of the total variance. The cluster analysis of the OCS revealed high variability, defined in two large groups: group 1 brought together OCS dominated by interchangeable $\mathrm{Al}^{3+}$ including 3 of the 12 OCSs and group 2 brought together 9 of the OCS, mainly defined by high content in soil organic matter (SOM). The variability showed was possibly influenced by liming and organic fertilization.

Key words: Interchangeable acidity, soil analysis, liming, organic matter, chemical properties.

\section{INTRODUCCIÓN}

Puente Abadía es una zona donde se ha venido produciendo, por muchas décadas, café de tipo orgánico (Colmenares et al. 2016). El café de tipo orgánico es un sistema de pro- 
ducción que, mediante el manejo racional de los recursos naturales, con baja o nula utilización de productos de síntesis química, mejora la calidad del café, mantiene o incrementa la fertilidad del suelo y la diversidad biológica (Rosas et al. 2008), concepto aplicable a sistemas de café bajo sombrío (Sadeghian, 2011). En los sistemas de café cultivados en Colombia existe una alta variabilidad de las condiciones químicas de los suelos de un sitio a otro; el clima, el suelo y el manejo son los factores más influyentes (Silva et al. 2008; Santos et al. 2015; Lince \& Sadeghian, 2015; Rodríguez-Garay et al. 2016). La fertilización y el encalado han sido señalados como las dos principales causas (Santos et al. 2015). De acuerdo con Camacho-Tamayo et al. (2010), la fertilidad de los suelos de la Orinoquia de Colombia se ha modificado, debido a la intervención agrícola, influyendo en la variabilidad, principalmente, de la materia orgánica $\mathrm{MO}$, el $\mathrm{pH}$ y el $\mathrm{Al}^{3+}$ intercambiable. En los sistemas cafeteros, el manejo de la fertilización, sin considerar la variabilidad espacial del suelo (Lince \& Sadeghian, 2015; Roger et al. 2014; Santos et al. 2015), da como resultado un uso poco eficiente de los nutrientes aplicados (Amaral et al. 2010). El conocimiento de la variabilidad espacial en suelos cafeteros es una herramienta que permite definir un manejo más específico de nutrientes para zonas más homogéneas (Silva et al. 2008; Rodríguez-Garay et al. 2016) y así optimizar recursos al reducir costos y reducir el impacto negativo sobre el ambiente (Lal, 2004); para el diagnóstico de la fertilidad del suelo, el análisis químico es la herramienta más usada (Camacho-Tamayo et al. 2010). Según Espinosa \& Gómez (2005), con éste, se evalúa la capacidad que posee un suelo para suministrar los nutrimentos que las plantas requieren para su normal crecimiento y desarrollo, con la finalidad de incrementar los rendimientos del grano (Sadeghian, 2011).

El objetivo de la presente investigación fue realizar un análisis multivariado de la fertilidad de los suelos en sistemas de café orgánico (SCO) de Puente Abadía, con el fin de que los resultados aporten sobre algunas limitantes químicas de los suelos para su producción y corresponda a un insumo importante para futuras investigaciones, dirigidas a establecer manejos de la fertilidad más acordes a las necesidades del cultivo, previos estudios de la extracción de nutrientes para el café, en esta zona.

\section{MATERIALES Y MÉTODOS}

Zona de estudio. Para esta investigación, se contemplaron sistemas de café orgánico (SCO), localizados en el Corregimiento de Puente Abadía, que pertenece a la ciudad de Villavicencio, ubicada entre las coordenadas geográficas

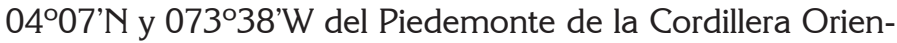
tal, al occidente del Departamento del Meta, en Colombia. De acuerdo con la clasificación de Köppen-Geiger, su clima es Am; presenta una precipitación media fluctuante, entre los $3.484,7 \mathrm{~mm}$ a $4.484 \mathrm{~mm}$; la temperatura varía de 25 a $30^{\circ} \mathrm{C}$, con una media de $25,5^{\circ} \mathrm{C}$; en febrero, se registran los valores más altos. La humedad relativa HR varía entre 76,1 a $82,1 \%$ y entre junio y julio, se registran HR $86 \%$ y el brillo solar es de 1.545 a 1.807 horas luz.año $^{-1}$ (Alcaldía de Villavicencio \& Secretaría de Medio Ambiente, 2006). Por su parte, Puente Abadía está ubicado a $28 \mathrm{~km}$ de Villavicencio, por vía destapada y está cercada por el río Guatiquía; los suelos se caracterizan por ser profundos, con texturas moderadamente finas a moderadamente gruesas, bien drenados, fuertemente ácidos, de fertilidad baja y susceptibles a la erosión, con predominancia de Ultisoles sobre Oxisoles (IGAC, 1995).

Selección de fincas. Entre el 2016 y 2017, se muestrearon 12 sistemas de café orgánico (SCO), distribuidos en 12 fincas, ubicadas a los $600 \mathrm{msnm}$, sembrados con café variedad Castillo de 5 años, bajo sombrío, principalmente del género Inga edulis y con una densidad de siembra de 1.600 plantas.ha $^{-1}$ (Colmenares et al. 2016); para todos los sitios, se muestrearon sistemas de café con las mismas condiciones agroecológicas y con el mismo manejo agronómico. En la preparación de los abonos orgánicos, se utiliza pulpa de café, mezclado con los estiércoles provenientes de las mismas fincas, similar a como se realiza en otras zonas de Colombia (Cardona-Calle \& Sadeghian, 2005). Según el análisis bromatológico realizado en el Laboratorio de Suelos de la Universidad de los Llanos, el compost elaborado en la zona, proporciona a los cultivos $4,5 \% \mathrm{~N}, 2 \% \mathrm{P}, 6,5 \% \mathrm{~K}$, $1,5 \% \mathrm{Ca}, 3,5 \% \mathrm{Mg}$ y elementos menores: $20 \% \mathrm{Mn}, 43,4 \% \mathrm{Cu}$, $22,8 \% Z n$ y $105 \% \mathrm{Fe}$. El compost, se aplica sin considerar el análisis de suelo, en cantidades que varían entre 2 a 5 t.ha ${ }^{-1}$, lo mismo sucede con la práctica de encalado, que es común y se realiza aplicando cal dolomita, en dosis que varían entre 2 a 3 t.ha ${ }^{-1}$. Se hacen aplicaciones foliares con biofertilizantes líquidos, preparados con productos orgánicos de la zona; el manejo fitosanitario de los cultivos, se realiza con extractos vegetales y se hace control biológico de la roya Hemileia vastatrix y la broca del café Hypothenemus hampei. Se denomina comúnmente "café de tipo orgánico", por el uso del sombrío (Colmenares et al. 2016).

Toma de muestras. En cada uno de los lotes de los 12 SCO identificados, que presentaban un área de $1.000 \mathrm{~m}^{2}$, se realizó un muestreo de tipo sistemático, en retícula de $10 \mathrm{~m} x$ $10 \mathrm{~m}$, para un total de 10 sitios y, en cada punto, se colectó una muestra compuesta, a partir de tres muestras simples, tomadas a una profundidad de $0-0,20 \mathrm{~m}$; el muestreo, se realizó en época de invierno. Las muestras, se enviaron al Laboratorio de Suelos de la Universidad de los Llanos, para análisis químicos.

Métodos analíticos. El pH, se midió con método potenciométrico; materia orgánica del suelo MOS (\%), con el método Walkley \& Black; aluminio intercambiable $\mathrm{Al}^{3+}\left(\mathrm{cmol}_{+}\right.$. 
$\mathrm{kg}^{-1}$ ), con $\mathrm{KCl} 1 \mathrm{~N}$ y espectrofotometría de absorción atómica; $\mathrm{Ca}, \mathrm{Mg}, \mathrm{K}$ y $\mathrm{Na}\left(\mathrm{cmol}_{+} \cdot \mathrm{kg}^{-1}\right)$, con el método de acetato de amonio $1 \mathrm{~N}$ a $\mathrm{pH} 7,0$; la CICE, por sumatoria de bases $\mathrm{y} \mathrm{Al}^{+}$intercambiable; $\mathrm{P}$ disponible $\left(\mathrm{mg} \mathrm{kg}^{-1}\right)$, mediante Bray II; contenidos de $\mathrm{Cu}, \mathrm{Fe}, \mathrm{Mn}$, Zn y B (mg.kg ${ }^{-1}$ ), con DTPA y espectrofotometría de absorción atómica. El S $\left(\mathrm{mg} \cdot \mathrm{kg}^{-1}\right)$, se determinó mediante fosfato monocalcico $0,008 \mathrm{M}$ y turbidimetría, siguiendo los métodos y los procedimientos estandarizados y recomendados por el IGAC (2006).

Análisis estadístico. Los resultados obtenidos en el laboratorio, se analizaron mediante estadística descriptiva, con el programa SPAD versión 5.0, con el cual, se determinó la media, la desviación estándar, el máximo, el mínimo y el coeficiente de variación (CV). El CV fue analizado con el criterio de Warrick \& Nielsen (1980), donde menores a $12 \%$ son de baja variabilidad; entre 12 y $60 \%$, de variabilidad media y superiores a $60 \%$, de alta variabilidad. Las correlaciones, se realizaron a través de la prueba de Pearson y la selección de relaciones entre elementos, como la interpretación de los valores mínimos y máximos, se basó en las consideraciones generales, para interpretar análisis de suelos (IGAC, 2000). El análisis de tipo multivariado compuesto por el análisis de componentes principales (ACPs) y el análisis de clúster, se realizó para SCO, a través del algoritmo de Ward, en el que se considera que la medida de disimilitud es la distancia euclidiana entre los atributos (Manly, 1997).

\section{RESULTADOS Y DISCUSIÓN}

Análisis de la estadística descriptiva. La media, la desviación estándar, el mínimo, el máximo y el coeficiente de variación (CV\%), se consignan en la tabla 1 . El pH tiene una media de 4,4 , que corresponde a suelos muy fuertemente ácidos, con un CV menor al 12\% (Tabla 1). Según Cox et al. (2006), el pH es una de las propiedades químicas del suelo que menos varía y su CV fluctúa de 2 a $15 \%$ y se debe a que se mide en escala logarítmica y, por consiguiente, se reduce la expresión de la variabilidad. En Ultisoles o Oxisoles es una característica intrínseca de la génesis del suelo y de las condiciones ambientales, principalmente, la precipitación (IGAC, 1995; Camacho-Tamayo et al. 2010). El Al ${ }^{3+}$ presentó una media de $0,95 \mathrm{cmol}_{+} \cdot \mathrm{kg}^{-1}$, que corresponde a bajos para suelos de la Orinoquia, a diferencia de los altos valores encontrados en Oxisoles, por Camacho-Tamayo et al. (2010). Este resultado está, posiblemente, asociado a la práctica intensiva de cal y compost, que ha permitido neutralizar, en parte, $\mathrm{el} \mathrm{Al}^{3+}$ intercambiable.

Tabla 1. Estadística descriptiva de las propiedades químicas del suelo en los SCO, Puente Abadía, Villavicencio.

\begin{tabular}{|c|c|c|c|c|c|c|}
\hline Propiedad & Unidad & $\bar{Y}$ & $\delta$ & $\mathbf{V}_{\mathbf{m}}$ & $\mathbf{V}_{\mathbf{M}}$ & CV\% \\
\hline $\mathrm{pH}$ & & 4,4 & 1,20 & 4,0 & 4,6 & 9,80 \\
\hline $\mathrm{MO}$ & $\%$ & 9,16 & 3,95 & 4,64 & 17,44 & 45,07 \\
\hline $\mathrm{Al}$ & $\mathrm{cmol}+\cdot \mathrm{kg}^{-1}$ & 0,95 & 0,58 & 0,25 & 1,95 & 63,89 \\
\hline $\mathrm{Ca}$ & $\mathrm{cmol}+. \mathrm{kg}^{-1}$ & 5,08 & 4,21 & 0,70 & 16,59 & 86,45 \\
\hline $\mathrm{Mg}$ & $\mathrm{cmol} \cdot . \mathrm{kg}^{-1}$ & 1,19 & 1,54 & 0,25 & 6,20 & 135,09 \\
\hline $\mathrm{K}$ & $\mathrm{cmol}+\cdot \mathrm{kg}^{-1}$ & 0,21 & 0,07 & 0,11 & 0,40 & 33,39 \\
\hline $\mathrm{Na}$ & $\mathrm{cmol}+\cdot \mathrm{kg}^{-1}$ & 0,11 & 0,05 & 0,06 & 0,23 & 48,89 \\
\hline $\mathrm{CICE}$ & $\mathrm{cmol}+\cdot \mathrm{kg}^{-1}$ & 7,56 & 2,21 & 7,43 & 8,40 & 9,75 \\
\hline $\mathrm{P}$ & $\mathrm{mg} \cdot \mathrm{kg}^{-1}$ & 17,13 & 7,51 & 5,70 & 28,50 & 45,75 \\
\hline $\mathrm{Cu}$ & $\mathrm{mg} \cdot \mathrm{kg}^{-1}$ & 8,73 & 5,09 & 4,00 & 24,30 & 60,92 \\
\hline $\mathrm{Mn}$ & $\mathrm{mg} \cdot \mathrm{kg}^{-1}$ & 116,67 & 44,89 & 75,25 & 233,12 & 40,18 \\
\hline $\mathrm{Zn}$ & $\mathrm{mg} \cdot \mathrm{kg}^{-1}$ & 16,73 & 14,88 & 2,65 & 49,37 & 92,86 \\
\hline $\mathrm{Fe}$ & $\mathrm{mg} \cdot \mathrm{kg}^{-1}$ & 110,20 & 10,2 & 98,45 & 115,78 & 9,20 \\
\hline $\mathrm{S}$ & $\mathrm{mg} \cdot \mathrm{kg}^{-1}$ & 11,70 & 8,48 & 3,13 & 36,18 & 75,69 \\
\hline $\mathrm{B}$ & $\mathrm{mg} \cdot \mathrm{kg}^{-1}$ & 1,21 & 1,03 & 0,47 & 4,02 & 88,72 \\
\hline
\end{tabular}

$\overline{\mathrm{Y}}=$ Media $\delta=$ Desviación estándar $\mathrm{V}_{\mathrm{m}}=$ Mínimo valor $\mathrm{V}_{\mathrm{M}}=$ Máximo valor $\mathrm{CV}=$ Coeficiente de variación. 
Casierra \& Aguilar (2007) afirman que la adición de materiales orgánicos al suelo corrige la toxicidad por $\mathrm{Al}^{3+}$, por su capacidad para formar complejos estables alumino-orgánicos, que reducen la fitotoxicidad del elemento. La neutralización del $\mathrm{Al}^{3+}$ intercambiable, mediante el encalado de los suelos, es una práctica común en suelos ácidos de Colombia (Castro \& Munévar, 2013); por lo tanto, en la tabla 1, se observan valores mínimos de $0,25 \mathrm{cmol}_{+} \cdot \mathrm{kg}^{-1}$, con máximos de $1,95 \mathrm{cmol}_{+} . \mathrm{kg}^{-1}$, para $\mathrm{Al}^{3+}$, típicos de Ultisoles o Oxisoles (IGAC, 1995), por lo que la variabilidad del $\mathrm{Al}^{3+}$ intercambiable en los SCO fue alta, con un CV de $63,89 \%$, similar a los reportados en Oxisoles de zonas cafeteras de Brasil, por Silva et al. (2008).

Según Castro \& Munévar (2013), el $\mathrm{Al}^{+3}$ es un limitante químico en la productividad de estos suelos y el problema se maneja con la adición de cales, que actúan como bases fuertes, que hidrolizan el agua y producen iones hidroxilos $\left(\mathrm{OH}^{-}\right)$, que neutralizan la acidez activa $\left(\mathrm{H}^{+}\right)$y precipitan el $\mathrm{Al}^{3+}$ en forma de oxihidróxidos $\left(\mathrm{Al}(\mathrm{OH})_{3}\right)$. La saturación de acidez es una medida del porcentaje del complejo de intercambio catiónico, que está ocupado por aluminio e hidrógeno y sería el mejor criterio para diagnosticar problemas de acidez (Castro \& Munévar, 2013). Las raíces afectadas por el Al exploran un volumen muy limitado del suelo y así se reduce su capacidad para tomar los nutrientes y el agua (Casierra \& Aguilar, 2007). En la tabla 1, en los SCO, la MOS muestra una media de $9,16 \%$, clasificado como alto, para suelos de clima cálidos (IGAC, 2000), resultado que es atribuido mayormente a SCO sobre Ultisoles (IGAC, 1995); la MO varió de 4,64 a 17,44\%, donde el valor mínimo es típico en Oxisoles (Camacho-Tamayo et al. 2010), presentando una desviación estándar de 3,95\%; el CV estuvo en un rango medio, de 45,07\%.
Según Silva et al. (2008), la moderada o alta variación en los contenidos de la MOS son consecuencia de varios factores, como la actividad microbiana, el tipo de cultivo, la cantidad y calidad de residuos vegetales y la fertilización. Cardona-Calle \& Sadeghian (2005) concluyeron que el retorno de material orgánico en cultivos de café con sombrío es 2,5 veces superior que en cultivos sin sombra. La MOS en los cafetales proviene, principalmente, de la hojarasca producida por las plantas de café y de los árboles de sombra, pero también de la fertilización orgánica (Sadeghian, 2011).

En la tabla 1, se indica que los SCO presentan contenidos medios de Ca y K, de 5,08 y 0,21 $\mathrm{cmol}_{+} . \mathrm{kg}^{-1}$ y de $\mathrm{Mg}$ y Na, de 1,19 y $0,11 \mathrm{cmol} \cdot \mathrm{kg}^{-1}$, que son bajos (IGAC, 2000). De ahí, que en la figura 1, se observa que en todos los SCO el $\mathrm{Ca}>\mathrm{Mg}>\mathrm{K}>\mathrm{Na}$. A pesar de las altas saturaciones de $\mathrm{Ca}$ en los suelos, éste es menos absorbido que el K, por las raíces del cultivo, sobre todo, en cafetales más viejos (Sadeghian et al. 2013). Los valores máximos de $\mathrm{Ca}$ y Mg en los SCO fueron de 16,59 y $6,20 \mathrm{cmol}_{+} \cdot \mathrm{kg}^{-1}$ y los mínimos de 0,70 y $0,25 \mathrm{cmol}_{+} \cdot \mathrm{kg}^{-1}$ (Tabla 1 ), clasificados como altos y bajos, respectivamente (IGAC, 2000). En suelos ácidos, los cationes de los elementos nutritivos son desplazados de los sitios de intercambio por el $\mathrm{Al}^{3+}$ intercambiable y, posteriormente, son lavados (Castro \& Munévar, 2013); $\mathrm{el} \mathrm{Al}^{3+}$ interfiere con la toma, el transporte y la utilización de las bases del suelo (Malavolta, 1993). De acuerdo con Cardona-Calle \& Sadeghian (2005), los contenidos de Ca y Mg adecuados para café bajo sombra, varían en los rangos de 0,48 a $10,30 \mathrm{cmol}+. \mathrm{kg}^{-1}$ y de 0,22 a $3,58 \mathrm{cmol}+. \mathrm{kg}^{-1}$; los encontrados están por encima de los rangos más altos. El café ha demostrado ser sensible a un bajo suministro del $\mathrm{Mg}$ en el suelo, encontrándose en cantidades relativamente altas en el fruto del café (Sadeghian et al. 2006; Sadeghian et al. 2013).

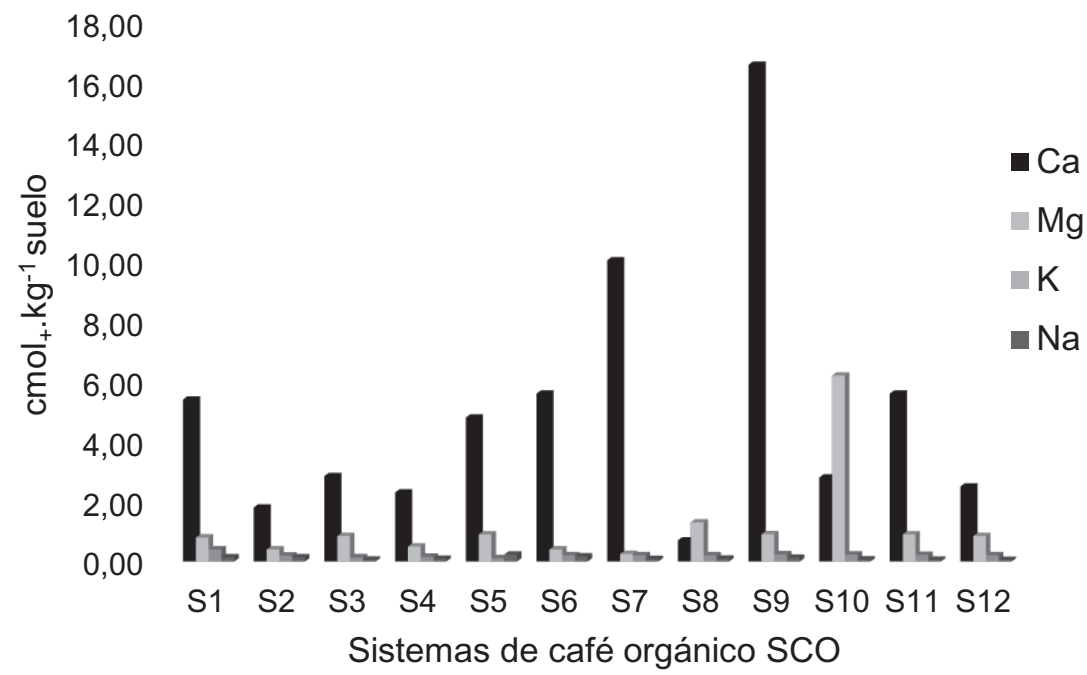

Figura 1. Contenidos de Ca, Mg, K y Na del suelo en SCO, Puente Abadía, Villavicencio. 
En Colombia, en café muy pocas veces se observan síntomas de deficiencia de Ca y S, pero sí de Mg (Sadeghian et al. 2013), acompañados de una defoliación, que puede ser severa, según la magnitud de la carencia del elemento (Malavolta, 1993); en la tabla 1, en los SCO, el K osciló entre 0,11 y $0,40 \mathrm{cmol}+. \mathrm{kg}^{-1}$. Sadeghian (2003) registró que, cuando el $\mathrm{K}$ en el suelo fue inferior a $0,2 \mathrm{cmol}_{+} \cdot \mathrm{kg}^{-1}$, se redujo el rendimiento en un $40 \%$; con 0,2 a $0,4 \mathrm{cmol}+. \mathrm{kg}^{-1}$, la reducción fue menor a $14 \%$ y para niveles mayores a $0,4 \mathrm{cmol}+. \mathrm{kg}^{-1}$, solo del $8 \%$, indicando que los SCO estudiados, que presentan un mínimo de $0,11 \mathrm{cmol}+. \mathrm{kg}^{-1}$ para $\mathrm{K}$, podrían tener limitaciones en la producción. La absorción del $\mathrm{K}$ no es lo suficientemente alta como para contrarrestar los efectos en el agotamiento de las reservas en las hojas viejas, requiriéndose siempre suficiencia de $\mathrm{K}$ en los suelos (Malavolta, 1993). En la tabla 1, en los SCO se presentaron altos CV para Mg y Ca $(86,45$ y $135,09 \%$, respectivamente) y medios, para Na y K (33,39 y 48,89\%, respectivamente). Los CV de Ca y de Mg fueron similares a los registrados por Rodríguez-Garay et al. (2016), en una zona cafetera y superiores a los hallados por Silva et al. (2008), en Oxisoles, de zonas cafeteras del Brasil; el CV de $\mathrm{K}$ fue menor al reportado por Rodríguez-Garay et al. (2016), que fue de 96\%. Esta alta variabilidad encontrada para Ca y Mg en los SCO, se puede deber al efecto residual del encalado (Castro \& Munévar, 2013); se sugiere, como adecuada, la relación $\mathrm{Ca} / \mathrm{Mg} / \mathrm{K}$ de 6:2:1, en zonas cafeteras de Colombia (Sadeghian, 2003). Las óptimas producciones de café consideran la extracción de nutrimentos de $\mathrm{N}$ y P, como las bases K, Ca y Mg (Sadeghian et al. 2006); en sistemas cafeteros fertilizados con $\mathrm{N}, \mathrm{K}$ y $\mathrm{P}$ es razonable prever que el Ca o el Mg resulten limitantes (Amaral et al. 2010). Por otra parte, en la tabla 1, en los SCO el Na del suelo no es una limitante, registrándose mínimos y máximos de 0,06 y $0,23 \mathrm{cmol}_{+} \cdot \mathrm{kg}^{-1}$ y una desviación estándar de $0,05 \mathrm{cmol}_{+}$. $\mathrm{kg}^{-1}$.

La CICE presentó una variabilidad baja $(9,75 \%)$, diferente a lo reportado por Rodríguez-Garay et al. (2016), que fue media. Los suelos de la Orinoquia de Colombia, se caracterizan por ser de carga variable, con altos contenidos de arcillas de baja actividad, como la caolinita y presencia de oxihidróxidos de $\mathrm{Al}$ y $\mathrm{Fe}$, que tienen una baja capacidad de intercambio catiónico, incidiendo en una baja retención de nutrientes (IGAC, 1995), coincidiendo con los mínimos y máximos encontrados, que fueron bajos $\left(7,43\right.$ y $8,40 \mathrm{cmol}+. \mathrm{kg}^{-1}$, respectivamente), en la tabla 1. En los SCO, la media para los contenidos de $\mathrm{P}$ disponible fue baja, de $17,13 \mathrm{mg} \cdot \mathrm{kg}^{-1}$, con una desviación estándar de 7,51 mg.kg ${ }^{-1}$ (Tabla 1), valor típico, tanto en Ultisoles como en Oxisoles (IGAC, 1995). Camacho-Tamayo et al. (2010), en Oxisoles, registraron altos contenidos de $\mathrm{P}$, atribuyéndolo a los efectos residuales de los fertilizantes. El P estuvo en un rango de bajo a medio, 5,70 a 28,50 mg. $\mathrm{kg}^{-1}$ y el CV fue medio de 45,75\% (Tabla 1), inferior al reportado por Roger et al. (2014), y al encontrado por
Rodríguez-Garay et al. (2016), de 58\%. Aunque este es un elemento que es poco usual que presente CV bajos, indica que, de manera general, en los SCO de la zona, la disponibilidad del $\mathrm{P}$ en suelos ácidos está condicionada a los procesos de fijación, no permitiendo que el P sea asimilable para las plantas (Malavolta, 1993; IGAC, 1995). Santos et al. (2015) halló que la distribución del $\mathrm{P}$ en el suelo fue heterogénea, variando de bajo a alto en el área de estudio; Andrade et al. (2009), por su parte, mencionan el efecto que pueden ejercer las micorrizas en sistemas de café bajo sombra, en la solubilización del mismo.

En la tabla 1, los micronutrientes estuvieron en el rango de bajos a altos menos en $\mathrm{Mn}$ y en $\mathrm{Fe}$, que fueron altos. El $\mathrm{Mn}$ presenta un CV de $40,18 \%$, considerado como medio y el $\mathrm{Fe}$, un CV bajo, de 9,20\%, ambos asociados, principalmente, con la acidez de los suelos (Castro \& Munévar, 2013), presentando mínimos y máximos altos de 75,25 y 233,12mg. $\mathrm{kg}^{-1}$ y 98.45 y $115,78 \mathrm{mg}_{\mathrm{kg}}{ }^{-1}$, respectivamente (Tabla 1 ). Los contenidos de Fe en los SCO, a niveles que pueden generar toxicidad (Malavolta, 1993), pueden estar asociados a los altos aportes del elemento en el compost; Mn se presentó muy por encima de los reportados por Cardona-Calle \& Sadeghian (2005), en Chinchiná (11,17mg. $\mathrm{kg}^{-1}$ ) y Guadalupe $\left(9,17 \mathrm{mg} \mathrm{kg}^{-1}\right)$, considerados adecuados para la producción de café. El $\mathrm{Mn}$ en altas concentraciones puede ser tóxico para el cultivo de café (Malavolta, 1993); al contrario, un bajo contenido de $\mathrm{Mn}$ en el suelo puede estar asociado a un alto contenido de $\mathrm{MO}$ en el suelo, el cual, disminuye considerablemente el Mn disponible (Malavolta, 1993). En la tabla 1, se observa, con respecto al S y B, que la media fue alta y baja, respectivamente, con un CV alto en ambos casos, de 75,69 y $88,72 \%$. El análisis foliar en sistemas de café es de gran ayuda para regular las aplicaciones de micronutrientes, de manera que se prevenga una posible deficiencia o toxicidad (Malavolta, 1993). En promedio, por cada $1.000 \mathrm{~kg}$ de café almendra, equivalentes a $1.250 \mathrm{~kg}$ de café pergamino seco (100 arrobas), se extraen 4,3kg de Ca, $2,3 \mathrm{~kg}$ de $M g$ y $1,2 \mathrm{~kg}$ de S (Sadeghian et al. 2006).

Correlaciones lineales. En la tabla 2, la MO presenta correlación positiva con $\mathrm{Ca}, \mathrm{P}$ y $\mathrm{Cu}$ y negativa con el contenido de Na y Zn, indicando que, en los SCO, la MOS podría ser un buen indicador también de $\mathrm{P}$ y Ca.

Según Castro \& Munévar (2013), los suelos de características ácidas ofrecen mejor respuesta a la aplicación de dosis bajas de cal y los contenidos de P aumenta, a medida que se incrementa el $\mathrm{pH}$, con el uso de materiales encalantes combinados. Por otra parte, el cafeto parece poseer una susceptibilidad especial a una baja concentración de $\mathrm{Zn}$ en el suelo (Martínez et al. 2011), cuando la MOS es baja. El Al ${ }^{3+}$ intercambiable presentó correlación positiva con $\mathrm{Mg}$, K, S y también con B y negativa con el Ca (Tabla 2). Cuando el por- 
Tabla 2. Tabla de Correlaciones de Pearson.

\begin{tabular}{|c|c|}
\hline Propiedad & Propiedad química con correlación $\left.\mathbf{~ R}^{2}\right)$ significativa \\
\hline $\mathrm{MO}$ & $\mathrm{Ca}(0,34), \mathrm{Na}(-0,46), \mathrm{P}(0,74), \mathrm{Cu}(0,35), \mathrm{Zn}(-0,52)$ \\
\hline $\mathrm{Al}$ & $\mathrm{Ca}(-0,33), \mathrm{Mg}(0,57), \mathrm{K}(0,49), \mathrm{S}(0,39), \mathrm{B}(0,52)$ \\
\hline $\mathrm{Ca}$ & $\mathrm{B}(-0,30)$ \\
\hline $\mathrm{Mg}$ & $\mathrm{Al}(0,57)$ \\
\hline $\mathrm{K}$ & $\mathrm{Al}(0,49)$ \\
\hline $\mathrm{Na}$ & $\mathrm{P}(-0,79) \mathrm{y} \mathrm{Zn}(0,79)$ \\
\hline $\mathrm{P}$ & $\mathrm{Cu}(-0,40), \mathrm{Zn}(-0,75)$ \\
\hline $\mathrm{Cu}$ & $\mathrm{Zn}(0,39), \mathrm{S}(0,72)$ \\
\hline $\mathrm{Mn}$ & $\mathrm{Na}(-0,37)$ \\
\hline $\mathrm{Zn}$ & $\mathrm{MO}(-0,52), \mathrm{Na}(0,79), \mathrm{P}(-0,75)$ \\
\hline $\mathrm{S}$ & $\mathrm{Al}(0,39), \mathrm{Mg}(-0,23), \mathrm{K}(0,73), \mathrm{Cu}(0,72)$ \\
\hline $\mathrm{B}$ & $\mathrm{Al}(0,52), \mathrm{Ca}(-0,30)$ \\
\hline
\end{tabular}

Adaptado de Rodríguez-Garay et al. (2016).

centaje de saturación de $\mathrm{Al}^{3+}$ intercambiable está por encima de $25 \%$, el $\mathrm{Al}^{3+}$ es el catión predominante en el complejo de cambio, bajando los niveles de las bases, principalmente el Ca (Castro \& Munévar, 2013); aplicaciones intensivas de cal, para neutralizar el $\mathrm{Al}$, hacen que el $\mathrm{B}$ sea menos absorbido por el cafeto, por lo que se puede mostrar deficiente (Furlani-Junior et al. 2004). En cafetero, el S es más requerido que el $\mathrm{P}$, cuando hay deficiencia de $\mathrm{S}$ y aunque no se observen síntomas, la florecida es normal, pero los frutos no cuajan (Malavolta, 1993). Una deficiencia de B se nota en las hojas adultas presentando suberización con presencia de corcho en la vena central y las secundarias (Rosolem \& Leite, 2007). $\mathrm{El} \mathrm{B}$ al igual que el $\mathrm{Ca}$, no se transloca a los puntos de crecimiento, es necesario un suministro constante de B para el desarrollo normal de las plantas (Santinato et al. 2016). En la tabla 2, el Mg correlacionó positivamente con el $\mathrm{Al}^{3+}$ intercambiable, el Na correlacionó negativamente con $\mathrm{P}$ y positivamente con Zn; el $\mathrm{P}$ mostró una correlación negativa con $\mathrm{Zn}$ y Cu, a su vez, éste correlacionó positivamente con el S. Según Rodríguez-Garay et al. (2016), en sistemas de café, se presentó una correlación positiva con $\mathrm{Cu}, \mathrm{Fe}$ y $\mathrm{Zn}$, además de ser el $\mathrm{Zn}$ el elemento que más se beneficia con incrementos en el pH, como se observó en las correlaciones del Fe y de P, respecto al Zn.

Análisis de componentes principales. En la tabla 3, se observa que, de las 12 propiedades químicas seleccionadas por el $\mathrm{CV}$, se determinaron cinco componentes principales $\mathrm{CPs}$, los cuales, presentan un autovalor superior a 1, en las características comunales (Manly, 1997) y que explican el $87,47 \%$ de la varianza total. En la figura 2, las dos primeras dimensiones del diagrama de dispersión espacial arrojado por el análisis de CPs explicaron el 51,06\% de la varianza de las propiedades químicas evaluadas en los SCO, de Puente Abadía. Las variables de mayor contribución al 28,55\% de la varianza, expresada por el primer componente principal, fueron la $\mathrm{MO}$ y el $\mathrm{P}$; se confirma la relación inversa de $\mathrm{Na}, \mathrm{Cu}$ y $\mathrm{Zn}$ con las otras variables. Además, en la tabla 3 , se observa el poco peso que el $\mathrm{Al}^{3+}$ intercambiable representa para el CP1. En el componente dos, que explica el 28,55\% de la varianza, las variables de mayor contribución y de manera

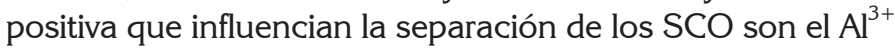
intercambiable, $\mathrm{K}$ y S y, en menor proporción, Cu y B, como se detalla en la tabla 3. En términos generales, las propiedades relacionadas con la acidez intercambiable del suelo son las que mejor representan el CP2. Para la producción de café y su relación con las propiedades del suelo, Castro-Tanzi et al. (2012) identificaron los contenidos de N, K, P y Mg, como los de mayor influencia. Rodríguez-Garay et al. (2016), en el CP1, identificaron los micronutrientes y las bases y en el $\mathrm{CP} 2$, el carbono orgánico y el Al, como las variables de más peso en la producción y calidad del café. En la tabla 3, se muestra que las variables que muestran la mayor comunalidad estimada son $\mathrm{Mg}, \mathrm{Ca}, \mathrm{K}, \mathrm{Al}$ y B, siendo los que más se correlacionan con el resto de atributos, lo cual, implica que las variables están muy bien representadas en el espacio de los factores, definiendo los clústeres de la figura 2; sin embargo, el Ca que presenta un valor superior a 0,97 en la comunalidad, en los dos primeros CPs, su valor no fue representativo, a diferencia del resultado de Rodríguez-Garay et al. (2016), donde el Ca presentó una correlación con el $\mathrm{CP} 1$ superior al $70 \%$. El uso excesivo de fertilizantes nitroge- 
nados (compost) con alta capacidad acidificante contribuye al deterioro de la fertilidad en Ultisoles, principalmente del Ca (Malavolta, 1993).

Análisis de agrupamiento jerárquico. El análisis de agrupación de las propiedades químicas de los SCO de Puente Abadía logró consolidar 4 clúster; el factor 1, definido por el
CP1, quedó determinado por los clústeres $3 / 4$ y 4/4, relativo a altos contenidos de MOS y P y que reunieron a la mayoría de los SCO, como se observa en la figura 2; las dos variables identificadas pueden estar relacionadas a una mayor fertilidad de los suelos de los SCO (IGAC, 1995). El factor 2 logró agrupar los clústeres $1 / 4$ y 2/4, conformados por los SCO S1, S6 y S5, que están formando un ángulo próximo a $45^{\circ}$,

Tabla 3. Comunalidades basadas en correlaciones.

\begin{tabular}{|c|c|c|c|c|c|c|}
\hline Variables & CP1 & CP2 & CP3 & CP4 & CP5 & Comunalidad \\
\hline MO & $\mathbf{0 , 7 3}$ & 0,25 & 0,03 & 0,45 & -0.06 & 0,82 \\
\hline $\mathrm{Al}$ & $-0,10$ & $\mathbf{0 , 7 9}$ & 0,49 & $-0,10$ & $-0,16$ & 0,91 \\
\hline $\mathrm{Ca}$ & 0,06 & $-0,17$ & $-0,44$ & 0,75 & $-0,42$ & 0,97 \\
\hline $\mathrm{Mg}$ & 0,22 & 0,30 & 0,54 & $-0,25$ & $-0,70$ & 0,98 \\
\hline $\mathrm{K}$ & $-0,22$ & $\mathbf{0 , 8 3}$ & $-0,36$ & 0,14 & $-0,19$ & 0,92 \\
\hline $\mathrm{Na}$ & $-\mathbf{0 , 8 4}$ & $-0,23$ & 0,18 & 0,31 & $-0,02$ & 0,88 \\
\hline $\mathrm{P}$ & $\mathbf{0 , 8 8}$ & 0,19 & $-0,06$ & 0,14 & 0,27 & 0,90 \\
\hline $\mathrm{Cu}$ & $-0,68$ & 0,53 & $-0,32$ & $-0,07$ & 0,01 & 0,85 \\
\hline $\mathrm{Mn}$ & 0,24 & 0,00 & $-0,53$ & $-0,58$ & 0,02 & 0,67 \\
\hline $\mathrm{Zn}$ & $-\mathbf{0 , 8 0}$ & $-0,38$ & 0,19 & 0,06 & $-0,01$ & 0,78 \\
\hline $\mathrm{S}$ & $-0,38$ & $\mathbf{0 , 7 3}$ & $-0,39$ & 0.05 & 0,21 & 0,87 \\
\hline $\mathrm{B}$ & $-0,03$ & 0,40 & 0,63 & 0,29 & 0,51 & 0,90 \\
\hline Autovalor & 3,42 & 2,70 & 1,84 & 1,41 & 1,11 & \\
\hline \% Total varianza & 28,55 & 22,51 & 15,39 & 11,75 & 9,26 & \\
\hline \% Acumulado varianza & 28,55 & 51,06 & 66,45 & 78,20 & 87,47 & \\
\hline
\end{tabular}

Valores $>0,7$ (valor absoluto) aparecen en negrilla.

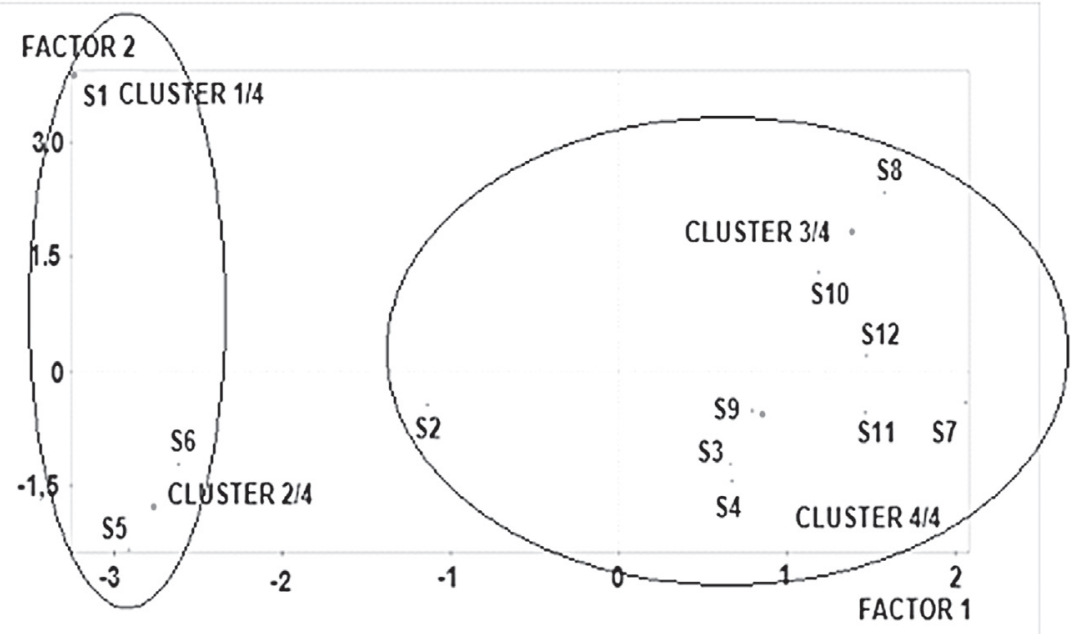

Figura 2. Conformación de clúster considerando los primeros dos factores o componentes principales CPs en SCO de Puente Abadía, Villavicencio. 
con cada eje (Figura 2), lo que no permite asociarlos con los SCO del factor 1, por sus características comunes, especificados por alto $\mathrm{Al}^{3+}$ intercambiable, bajo $\mathrm{K}$ y alto $\mathrm{S}$, de mayor peso en el CP2 (Tabla 3). $\mathrm{El} \mathrm{Al}^{3+}$ intercambiable incide en detrimento del K (Malavolta, 1993), sería más claro el efecto si estuviesen relacionados en términos de saturación (Castro \& Munévar, 2013). El análisis de clústeres, relacionados en la figura 2, se detectaron dos tipologías de SCO, las cuales, se puede apreciar en el respectivo dendograma (Figura 3). La primera tipología representa el 33,3\% de los SCO analizados, que se caracterizan por altos niveles de $\mathrm{Al}^{+3}$ y bajo $\mathrm{K}$, indicando una mayor similitud entre los SCO S5 y S6 y, a su vez, fueron distantes del S1, de mayor acidez intercambiable y bajo K; similarmente, Camacho-Tamayo et al. (2010), observaron, en la conformación de los grupos, la relación estrecha entre el $\mathrm{Al}^{3+}$ intercambiable con las bases. El manejo de la fertilidad de los SCO de este grupo deberá incluir recomendaciones de encalado, acordes a la necesidad de neutralización de este elemento (Castro \& Munévar, 2013). En la figura 3, la segunda tipología, se caracteriza por el $66,6 \%$ de los SCO, que giran en torno a altos contenidos de MOS y medios de P, donde todos los SCO de este grupo guardan similitud $(P>0.01)$. Los altos niveles de MOS favorecen, desde una perspectiva sostenible, la producción de café de los SCO de este grupo, en dicha región; sin embargo, es necesario revaluarla y hacer otras investigaciones tendientes a su caracterización y así definir si se está cumpliendo con los requerimientos de NPK y micronutrientes necesarios para el cultivo. En el análisis multivariado, los agrupamientos se pueden delimitar como zonas de manejo, por la similitud que presentan los puntos (Rodríguez-Garay et al. 2016).

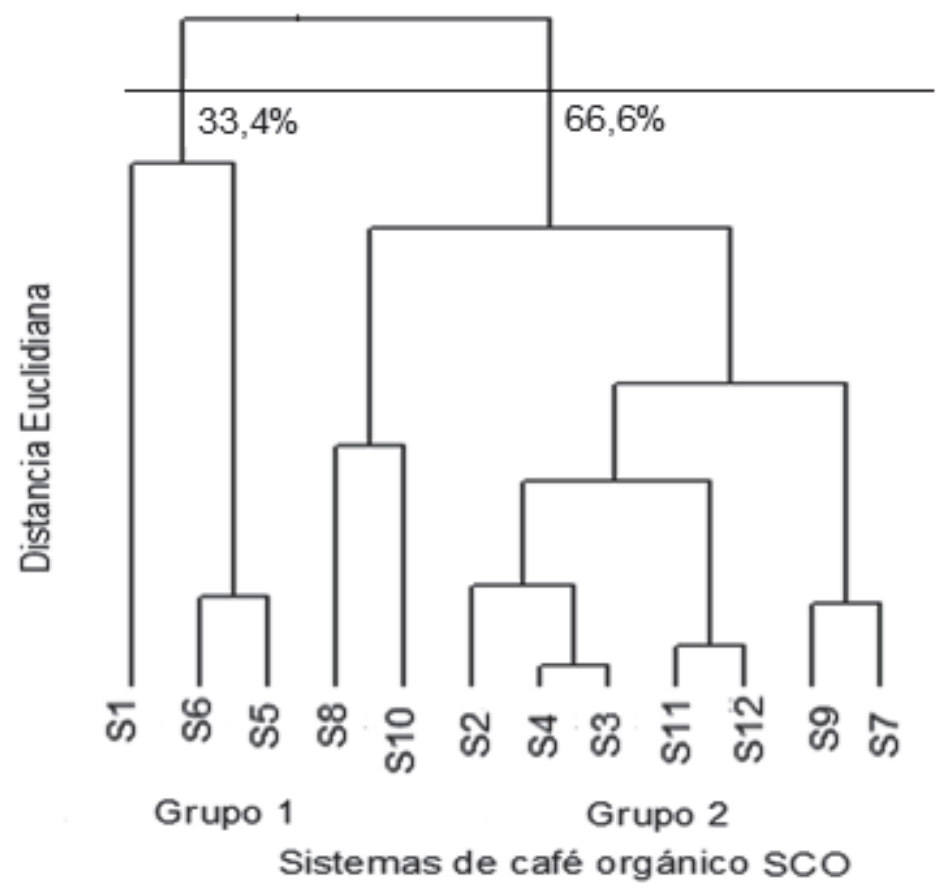

Figura 3. Dendograma para puntos de muestreo en SCO de Puente Abadía, Villavicencio.

Agradecimientos: Los autores agradecen a los productores de Puente Abadía, por su acompañamiento en esta investigación. Conflicto de intereses: El manuscrito fue preparado y revisado con la participación de todos los autores, quienes declaramos que no existe conflicto de intereses que ponga en riesgo la validez de los resultados presentados. Financiación: Esta investigación fue financiada por el proyecto "Fortalecimiento de la transferencia de tecnología agropecuaria a la comunidad, vereda Puente Abadía, municipio de Villavicencio", perteneciente a la Facultad de Ciencias Agropecuarias y Recursos Naturales, de la Universidad de los Llanos.

\section{BIBLIOGRAFÍA}

1. ALCALDÍA DE VILLAVICENCIO Y SECRETARÍA DE MEDIO AMBIENTE. 2006. Sistema de Gestión Ambiental "SIGAM" Municipio de Villavicencio. Perfil Ambiental y Plan de Acción. Informe Final Villavicencio. Villavicencio, Colombia: CORPOLLANOS. 129p.

2. AMARAL, J.F.T.D.; MARTINEZ, H.E.P.; LAVIOLA, B.G.; FERNANDES FILHO, E.I.; CRUZ, C.D. 2010. Bean production efficiency and relative allocation of nu- 
trients of four coffee varieties. Rev. Ceres. 57:253262.

3. ANDRADE, S.; MAZZAFERA, P.; SCHIAVINATO, M.; SILVEIRA, A. 2009. Arbuscular mycorrhizal association in coffee. The Journal of Agricultural Science. 147(2):105-115.

4. CAMACHO-TAMAYO, J.H.; LUENGAS-GOMEZ, C.; LEIVA, F.R. 2010. Análisis multivariado de propiedades químicas en Oxisoles con diferentes niveles de intervención agrícola. Acta Agronómica. 59(3):273-284.

5. CARDONA-CALLE, D.A.; SADEGHIAN, S. 2005. Evaluación de propiedades físicas y químicas de suelos establecidos con café bajo sombra y a plena exposición solar. Cenicafé. 56(4):348-364.

6. CASIERRA, F.; AGUILAR, O. 2007. Estrés por aluminio en plantas: reacciones en el suelo, síntomas en vegetales y posibilidades de corrección. Rev. Col. de Ciencias Hortícolas. 1(2):246-257.

7. CASTRO-TANZI, S.; DIETSCH, T.; URENA, N.; VINDAS, L.; CHANDLER, M. 2012. Analysis of management and site factors to improve the sustainability of smallholder coffee production in Tarrazú, Costa Rica. Agric. Ecosyst. Environ.155:172-181.

8. CASTRO, J.; MUNÉVAR, O. 2013. Mejoramiento químico de suelos ácidos mediante el uso combinado de materiales encalantes. Rev. U.D.C.A Act. \& Div. Cient. 16(2):409-416.

9. COLMENARES P., C.H.; SILVA, A.; MOGOLLÓN, Á.M. 2016. Impacts of different coffee systems on soil microbial populations at different altitudes in Villavicencio (Colombia). Agron. Colomb. 34(2):285-291.

10. COX, M.S.; GERARD, P.D.; MELINDA, A.J. 2006. Selected soil properties variability and their relationships with yield in three Mississippi fields. Soil Sci. 171:541551.

11. ESPINOSA, J.; GÓMEZ, M. 2005. Conceptos utilizados para diagnosticar integralmente la fertilidad del suelo mediante el uso de indicadores. Rev. U.D.C.A Act. \& Div. Cient. 8(2):23-32.

12. FURLANI-JÚNIOR, E.; ALVES, C.C.; LAZARINI, E.; FERRARI, S. 2004. Aplicação de calcário e boro em cafeeiro (Coffea arábica L.). Cultura Agronômica. 13:1-25.
13. INSTITUTO GEOGRÁFICO AGUSTÍN CODAZZI -IGAC-. 1995. Suelos de Colombia. Ed. IGAC, Subdirección Agrológica. Bogotá (Colombia). 632p.

14. INSTITUTO GEOGRÁFICO AGUSTÍN CODAZZI -IGAC-. 2006. Métodos analíticos de laboratorio de suelos. Ed. IGAC (Colombia). 648p.

15. INSTITUTO GEOGRÁFICO AGUSTÍN CODAZZI -IGAC-. 2000. Consideraciones generales para interpretar análisis de suelos. Subdirección de agrología. Bogotá, Colombia: IGAC. 324p.

16. LAL, R. 2004. Soil carbon sequestration impacts on global climate change and food security. Science. 304(5677):1623-1627.

17. LINCE, L.A.; SADEGHIAN, S. 2015. Producción de café (Coffea arabica L.) en respuesta al manejo específico de la fertilidad del suelo. Revista de Investigación Agraria y Ambiental. 6(2):29-30.

18. MALAVOLTA, E. 1993. Nutrição mineral e adubação do cafeeiro. São Paulo: Agronômica Ceres. 210p.

19. MANLY, B.F.J. 1997. Multivariate statistical methods. Second Ed. Chapman \& Hall (London). 216p.

20. MARTINEZ, H.E.P.; ZABINI, A.V.; CRUZ, C.D.; PEREIRA, A.A.; FINGER, F.L. 2011. Differential tolerance to zinc deficiency in coffee-plant progenies. J. Plant Nutrition. 34(11):1654-1674.

21. RODRÍGUEZ-GARAY, F.A.; CAMACHO-TAMAYO, J.H.; RUBIANO-SANABRIA, Y. 2016. Variabilidad espacial de los atributos químicos del suelo en el rendimiento y calidad de café. Corpoica Cienc. Tecnol. Agrop. 17(2):237-254.

22. ROGER, A.; LIBOHOVA, Z.; ROSSIER, N.; JOOST, S.; MALTAS, A.; FROSSARD, E.; SINAJ, S. 2014. Spatial variability of soil phosphorus in the Fribourg canton, Switzerland. Geoderma. 217-218:26-36.

23. ROSAS, J.; ESCAMILLA, E.; RUIZ, O. 2008. Relación de los nutrimentos del suelo con las características físicas y sensoriales del café orgánico. Terra Latinoamericana. 26(4):375-384.

24. ROSOLEM, C.A.; LEITE, V.M. 2007. Coffee leaf and stem anatomy under boron deficiency. Rev. Bras. Ciênc. Solo. 31(3):477-483. 
25. SADEGHIAN KH., S. 2003. Efecto de la fertilización con nitrógeno, fósforo, potasio y magnesio sobre las propiedades químicas de suelos cultivados en café. Cenicafé. 54(3):242-257.

26. SADEGHIAN, S.; GARCÍA, J.C.; MONTOYA, E.C. 2006. Respuesta del cafeto a la fertilización con $\mathrm{N}, \mathrm{P}, \mathrm{K}$ y $\mathrm{Mg}$ en dos fincas del departamento del Quindío. Cenicafé. 56(4):58-69.

27. SADEGHIAN, S. 2011. Respuesta de cafetales al sol y bajo semisombra a nitrógeno y su relación con la materia orgánica del suelo. Rev. Fac. Nal. Agr. 64(1):5781-5791.

28. SADEGHIAN K., S.; MEJÍA M., B.; GONZÁLEZ, O.H. 2013. Acumulación de calcio, magnesio y azufre en los frutos de café (Coffea arabica L.) Variedad Castillo®. Cenicafé. 64(1):7-18.

29. SANTINATO, F.; TAVARES, T.D.O.; PRADO, R.D.M.; CAIONE, G.; SILVA, V.D.A.; SANTINATO, R. 2016.
Boron doses applied to soil during coffee development. Comunicata Scientiae. 7(1):49-55.

30. SANTOS, E.O.J; GONTIJO, I.; SILVA, M.B.; NETO, A.P.D. 2015. Variabilidade espacial de macronutrientes em uma lavoura de café conilon no Norte do Espírito Santo. Rev. Ciênc. Agron. 46(3):469-476.

31. SILVA, F.M.; SOUSA, Z.M.; FIGUEIREDO, C.A.P.; SOUSA, L.H.S.; OLIVEIRA, E. 2008. Variabilidade espacial de atributos químicos e produtividade da cultura do café em duas safras agrícolas. Ciênc. Agrotec. 32(1):231-241.

32. WARRICK, A.W.; NIELSEN, D.R. 1980. Spatial variability of soil physical7 de 2017 properties in the field. In: Hillel, D. (ed.) Applications of soil physics. New York, Academic Press. 350p.

Recibido: Marzo 30 de 2017

Aceptado: Septiembre 7 de 2017

\section{Cómo citar:}

Silva-Parra, A.; Colmenares-Parra, C.; Álvarez-Alarcón, J. 2017. Análisis multivariado de la fertilidad de los suelos en sistemas de café orgánico en Puente Abadía Villavicencio. Rev. U.D.C.A Act. \& Div. Cient. 20(2): 289-298. 